\title{
Design of a test facility for probe calibration
}

\author{
Jan Šimák ${ }^{1, a}$ \\ ${ }^{1}$ Aerospace Research and Test Establishment, Department of Aerodynamics, Beranových 130, 19905 Prague - Letňany, Czech \\ Republic
}

\begin{abstract}
A possibility to easily calibrate probes for flow field measurements is always welcome. From this reason, a design of a test facility for probe calibration was made. The probes will be calibrated in a free jet of known properties, which is created by an exchangeable nozzle to cover a wide range of Mach numbers up to Mach 2. The most important is to create a homogeneous flow across the test section. This is accomplished by a precise design of the nozzles carried out by numerical tools. The convergent nozzle part is common for all subsonic flow regimes while the divergent part (forming a de Laval nozzle) is suited for a specific supersonic Mach number. These parts are designed using the method of characteristics. Numerical simulations performed by a CFD code show a feasibility and quality of the proposed test facility.
\end{abstract}

\section{Introduction}

Evaluation of experiments from internal aerodynamics is still dependent on measurements by pressure probes. However, the probes cannot measure the flow velocity and angles directly. Every probe can be slightly different due to manufacturing inaccuracy or a wear level and thus a calibration needs to be done before using. To calibrate a probe we need a flow of well known properties within a wide range of Mach and Reynolds number. Having such a device can significantly shorten the time needed to perform an experiment.

\section{Design of the device}

The calibrating device is intended to be a part of a closed circuit wind tunnel. A round nozzle creating a stream of air with required parameters will be installed inside a testing chamber. For subsonic regimes, the nozzle has a convergent shape. For supersonic regimes, a divergent extension is attached to form a de Laval type nozzle. The subsonic (convergent) part remains the same for the whole range of assumed velocities. The supersonic extension has an individual shape for each specific outgoing Mach number.

On the inlet of the compressed air into the nozzle, there are a honeycomb and screens to reduce the inlet turbulence and to homogenize the flow. The probe will be inserted into the free jet behind the nozzle in the place of the expected homogeneous flow (figure 1).

\subsection{Subsonic nozzle}

The inlet diameter of the convergent nozzle is $d_{i n}=$ $500 \mathrm{~mm}$ and the exit diameter is $d_{\text {out }}=100 \mathrm{~mm}$. Thus the

\footnotetext{
${ }^{\mathrm{a} C}$ Corresponding author: simak@vzlu.cz
}

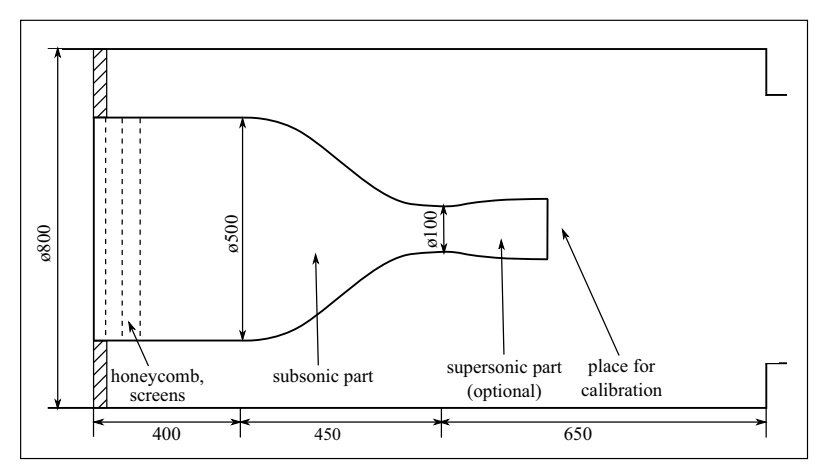

Figure 1: Scheme of the calibrating device with the nozzle.

compression ratio is $\mathrm{CR}=25$. The shape of the subsonic nozzle is described with a polynomial of the 5 th degree [? ],

$$
r(x)=r_{\text {in }}-\left(r_{\text {in }}-r_{\text {out }}\right)\left[6(x / L)^{5}-15(x / L)^{4}+10(x / L)^{3}\right],
$$

where $r$ is the radius at the distance $x$ from the beginning, $r_{\text {in }}$ and $r_{\text {out }}$ are the inlet and exit radius and $L$ is the nozzle length.

The exit Mach number is determined by a ratio of the total pressure before the nozzle $p_{i n, c}$ and the static pressure $p_{\text {out }}$ behind the nozzle exit. In the case of a subsonic flow, this pressure is equal to the chamber static pressure $p_{b}$. The Mach number can be expressed for an isentropic flow as

$$
M_{\text {out }}=\sqrt{\frac{2}{\kappa-1}\left[\left(\frac{p_{\text {in }, c}}{p_{\text {out }}}\right)^{(\kappa-1) / \kappa}-1\right]} .
$$

In the numerical simulations, the pressure $p_{\text {out }}$ is set to $102.315 \mathrm{kPa}$ and the total temperature $T_{i n, c}=300 \mathrm{~K}$ and 
Table 1: Quality of laminar flow for various nozzle length, $M_{\text {out }}=0.5$ (boundary layer properties, Reynolds number based on momentum thickness, uniformity).

\begin{tabular}{|c|c|c|c|c|c|c|}
\hline$L / d_{\text {in }}$ & $\delta[\mathrm{mm}]$ & $\delta^{*}[\mathrm{~mm}]$ & $\theta[\mathrm{mm}]$ & $H$ & $\operatorname{Re}_{\theta}$ & uniformity [\%] \\
\hline 0.4 & 0.615 & 0.365 & $4.322 \mathrm{e}-2$ & 8.439 & 494 & 3.020 \\
0.8 & 0.444 & 0.153 & $5.326 \mathrm{e}-2$ & 2.875 & 610 & 0.650 \\
0.9 & 0.459 & 0.156 & $5.543 \mathrm{e}-2$ & 2.809 & 635 & 0.484 \\
1.4 & 0.544 & 0.178 & $6.599 \mathrm{e}-2$ & 2.690 & 756 & 0.161 \\
\hline
\end{tabular}

Table 2: Pressure ratio, mass and volumetric flow rates.

\begin{tabular}{|c||c|c|c|c|c|c|}
\hline$M_{\text {out }}$ & 0.2 & 0.5 & 0.9 & 1.1 & 1.5 & 2.0 \\
\hline$p_{\text {in,c }} / p_{\text {out }}$ & 1.028 & 1.186 & 1.691 & 2.135 & 3.671 & 7.824 \\
$Q_{m}[\mathrm{~kg} / \mathrm{s}]$ & 0.641 & 1.644 & 3.127 & 3.990 & 6.865 & 14.644 \\
$Q_{V}\left[\mathrm{~m}^{3} / \mathrm{s}\right]$ & 0.525 & 1.166 & 1.556 & 1.573 & 1.574 & 1.575 \\
\hline
\end{tabular}

no heat flow through walls is assumed. For simplicity, the results presented below are obtained for an outflow into a free space and the influence of the restricted space of the chamber is discussed later.

We expect a low inlet velocity up to $8 \mathrm{~m} / \mathrm{s}$ and the incoming turbulent structures are reduced with the set of a honeycomb and screens. The high CR and the resulting pressure gradient increase the chance of a relaminarization of a turbulent boundary layer inside the convergent nozzle. From this reasons, it is justifiable to solve the flow in the subsonic nozzle part as a laminar problem.

The length of the nozzle was selected according to preliminary results as $L=450 \mathrm{~mm}$ (ratio $L / d_{i n}=0.9$ ). This length is a compromise between a growth of a boundary layer (and increased losses) and a decrease of a uniformity of the exit stream. The uniformity is evaluated as a standard deviation of the Mach number outside the boundary layer. The results for $M_{\text {out }}=0.5$ indicate, that the shortest variant causes separation of the flow in the throat. The longer variants do not show this undesirable condition. The other critical region for a possible flow separation is the beginning of the contraction. The flow separates there (and becomes attached later) for $L / d_{\text {in }}<0.8$ at $M_{\text {out }}=0.5$. With lower $M_{\text {out }}$, the sufficient length rises. However, the influence of this separation is not so strong due to the favourable pressure gradient.

\subsection{Numerical method - method of characteristics}

The supersonic nozzle is determined by the method of characteristics (MOC), which is a very popular tool for solving problems of supersonic flows. It is based on favourable properties of equations which describe a supersonic flow field. We assume a compressible, inviscid and stationary flow of a perfect gas with a negligible vorticity and heat transfers. That means an isentropic flow. The problem can be considered as axisymmetric and so a formulation in cylindrical coordinates is natural. Further we assume that the domain where the flow is solved (i.e. inside of the supersonic nozzle) has suitable properties, namely is bounded and simply connected, and the state variables $\left(u, v_{r}, v_{\omega}\right), p$ and $\varrho$ have continuous first derivatives. Hence there exists a velocity potential and the flow can be described by a quasilinear partial differential equation of a second order, which is hyperbolic for supersonic velocities. From this equation, we derive a system of equations for the so called characteristics (see [? ],[? ] chap. 13). A characteristic is a line (or surface in three dimensions), along which the first derivatives of state variables are indeterminate although the state variables itself are continuous. Along a characteristic we can transform the governing partial differential equation into an ordinary differential equation.

Right characteristic $C^{+}$:

$$
\begin{array}{r}
\frac{\mathrm{d} \varphi}{\mathrm{d} x}+F(M) \frac{\mathrm{d} M}{\mathrm{~d} x}-Q^{+}(M, \varphi) \frac{1}{r}=0, \\
\frac{\mathrm{d} r}{\mathrm{~d} x}=\tan (\varphi-\mu) .
\end{array}
$$

Left characteristic $C^{-}$:

$$
\begin{array}{r}
\frac{\mathrm{d} \varphi}{\mathrm{d} x}-F(M) \frac{\mathrm{d} M}{\mathrm{~d} x}+Q^{-}(M, \varphi) \frac{1}{r}=0 \\
\frac{\mathrm{d} r}{\mathrm{~d} x}=\tan (\varphi+\mu),
\end{array}
$$

where $M$ is the Mach number, $\varphi$ the direction of the flow, $\mu=\arcsin (1 / M)$ the Mach angle, $\gamma$ the adiabatic constant,

$$
\begin{array}{r}
F(M)=\frac{\sqrt{M^{2}-1}}{M\left(1+\frac{\gamma-1}{2} M^{2}\right)}, \\
Q^{+}(M, \varphi)=\frac{\tan \varphi}{\sqrt{M^{2}-1}+\tan \varphi}, \\
Q^{-}(M, \varphi)=\frac{\tan \varphi}{\sqrt{M^{2}-1}-\tan \varphi} .
\end{array}
$$

That means, the direction of characteristics is the same as the direction of the Mach waves propagation.

Every point of the computational domain can be considered as an intersection of the corresponding left and right characteristic. Using the notation from the figure 2 
we can evaluate unknown variables at the point 3 from the known values at points 1 a 2 :

$$
\begin{array}{r}
\varphi_{3}-\varphi_{2}+F\left(M_{23}\right)\left(M_{3}-M_{2}\right)-Q^{+}\left(M_{23}, \varphi_{23}\right) \frac{x_{3}-x_{2}}{r_{23}}=0, \\
\varphi_{3}-\varphi_{1}-F\left(M_{13}\right)\left(M_{3}-M_{1}\right)+Q^{-}\left(M_{13}, \varphi_{13}\right) \frac{x_{3}-x_{1}}{r_{13}}=0, \\
(11) \\
r_{3}-r_{2}-\left(x_{3}-x_{2}\right) \tan \left(\varphi_{23}-\mu_{23}\right)=0, \\
r_{3}-r_{1}-\left(x_{3}-x_{1}\right) \tan \left(\varphi_{13}+\mu_{13}\right)=0,
\end{array}
$$

where the subscript $i j$ denotes an average of values at points $i$ and $j$. The characteristics are thus approximated by piecewise linear curves. This system is perfectly valid inside the domain and, using the symmetry, near the axis as well.

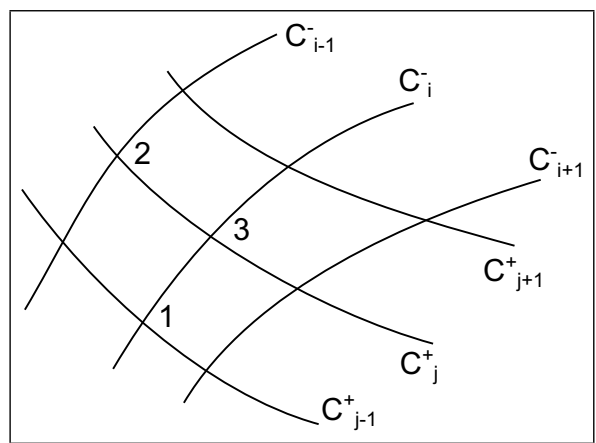

Figure 2: Grid of characteristics inside the computational domain.

The nonlinear system can be rewritten into a vector form

$$
S(q)=0, q=\left(x_{3}, r_{3}, M_{3}, \varphi_{3}\right)^{T},
$$

and solved by a damped Newton method

$$
\frac{\mathrm{D} S\left(q_{k}\right)}{\mathrm{D} q}\left(q_{k+1}-q_{k}\right)=-\alpha S\left(q_{k}\right)
$$

with $0<\alpha \leq 1$ a damping factor and $q_{0}$ a suitable initial condition. In this case we chose $q_{0}=\left(x_{12}, r_{12}, M_{12}, \varphi_{12}\right)^{T}$. If the grid is sufficiently dense, the initial condition is close to the solution and the method rapidly converges. The Jacobian can be expressed analytically (see [? ]).

The situation is slightly different when the point 3 is on a wall (figure 3). Assume some point from the wall, then the flow direction follows from the geometry (i.e. $x_{3}, r_{3}$ and $\varphi_{3}$ are known). The only unknown is the Mach number $M_{3}$. Suppose we know some previous characteristic $C_{j-1}^{+}$. We construct a characteristic $C_{p}^{-}$going through the point 3 . The intersection of $C_{j-1}^{+}$and $C_{p}^{-}$, denoted as 1 , is a linear combination of points $\overline{1}$ and $\overline{2}$ with a parameter $t \in \mathbb{R}$.

The equations (11) and (13) can be therefore rewritten as

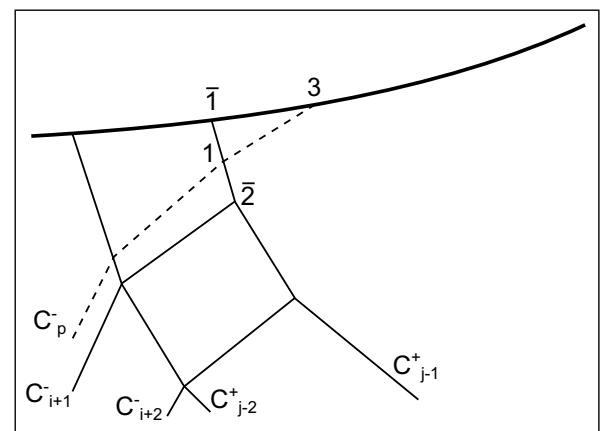

Figure 3: Characteristic on a wall.

$$
\begin{aligned}
\varphi_{3}-\varphi_{1}(t)- & F\left(M_{13}(t)\right)\left(M_{3}-M_{1}(t)\right) \\
& +Q^{-}\left(M_{13}(t), \varphi_{13}(t)\right) \frac{x_{3}-x_{1}(t)}{r_{13}(t)}=0, \\
r_{3}-r_{1}(t)-( & \left.x_{3}-x_{1}(t)\right) \tan \left(\varphi_{13}(t)+\mu_{13}(t)\right)=0
\end{aligned}
$$

for two unknowns $M_{3}$ and $t$.

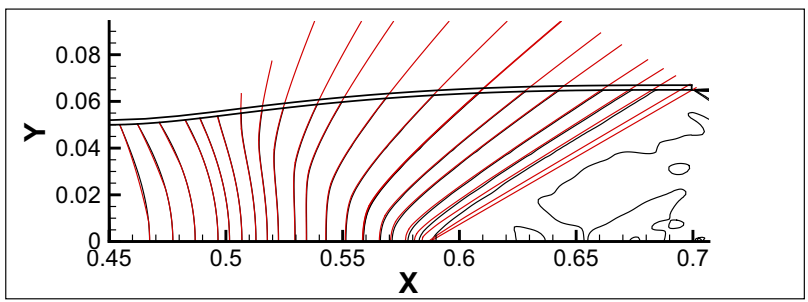

Figure 4: Comparison of Mach number distribution obtained by MOC (red) and inviscid CFD (black). Nozzle geometry for $M_{\text {out }}=2$.

The comparison of flow fields obtained by MOC and CFD shows good agreement (figure 4).

\subsection{Getting the supersonic nozzle shape}

The supersonic exit Mach number is determined by a ratio of the throat and exit area $A_{t} / A_{\text {out }}$,

$$
\frac{A_{t}}{A_{\text {out }}}=M_{\text {out }}\left[\frac{2}{\gamma+1}\left(1+\frac{\gamma-1}{2} M_{\text {out }}^{2}\right)\right]^{-\frac{\gamma+1}{2(\gamma-1)}} .
$$

With the knowledge of the throat radius $r_{t}=50 \mathrm{~mm}$ and the target $M_{\text {out }}$ it is possible to determine the exit radius $r_{\text {out }, \text { id }}$ for an isentropic flow. However, boundary layers have an impact on the optimal nozzle shape in a real flow. To preserve the exit area $A_{\text {out }}$, the real exit radius $r_{\text {out } v i s}>$ $r_{\text {out }, \text { id }}$ must be found. This correction will be discussed later.

Four main regions can be identified inside the nozzle (figure 5). It is a subsonic region before the throat, a region of an initial expansion, the straightening of the flow and finally a region of homogeneous flow. Some authors divide the nozzle into more parts, which may ensure more 
flexibility of the design (e.g. [? ? ]) but we consider the mentioned partitioning sufficient. The origin of the coordinate system is located in the centre of the geometrical throat.

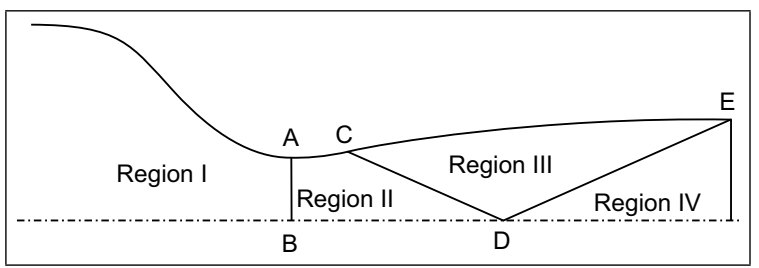

Figure 5: Principal regions of a supersonic nozzle.

The region $\mathrm{I}$ is responsible for an acceleration of the flow to a sonic speed and its shape is not so important for the quality of the exit flow unless it causes a flow separation near walls. The convergent geometry (1) used as the subsonic nozzle seems to be well designed to be also used as a part of the supersonic nozzle.

\section{Region II}

The region II is an area between the sonic line $\mathrm{AB}$ and a right characteristic through the point $\mathrm{D}$, which is a point on the axis where the demanded $M_{\text {out }}$ is reached. The position of the point $\mathrm{D}$ is unknown beforehand and must be obtained from the calculations.

We assume for simplicity that the sonic line is straight, i.e. the line segment $\mathrm{AB}$, and the flow on that line is parallel to the axis. This can be achieved by a nozzle with zero curvature of the throat. Otherwise, the sonic line is curved and the flow structure down the throat is more complex. However, if the curvature, compared to the throat radius, is not high as in our case, it is possible to make this assumption without an excessive loss of precision.

We need to set appropriate boundary conditions to be able to start with the creation of the characteristics grid. Usually, these include values on the sonic line, which follows from our assumption, and values either on the nozzle wall or on the axis. The presented method uses the first option, a prescribed shape of the initial expansion. The choice of the geometry is not determined uniquely and it influences the resulting shape of the region III contour.

The gridpoints are evaluated step by step starting from the point 1 (see Figure 6). We know values on the sonic line and the coordinates of points on the nozzle wall together with the tangent. We will construct the right characteristics $C_{j}^{+}, j=1, \ldots, n$. The process always begins on the wall, using the equations (16) and (17) to determine the value of the Mach number. After that, intersections with the left characteristics $C_{i}^{-}, i=1, \ldots, j$ are found by solving the system (14). In this way, we get a characteristics grid in the area of the region II. During the grid construction it may happen that some of the left characteristics intersect the nozzle wall. This indicates a reflection of expansion waves, now weaker, back into the nozzle.

The starting points of right characteristics are chosen such that the grid sufficiently covers the given area, with

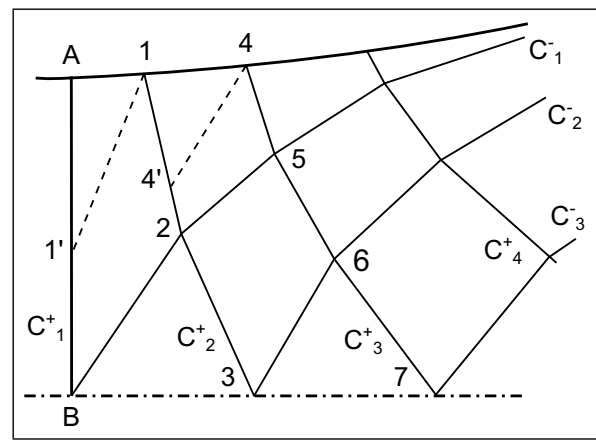

Figure 6: Process of grid construction in region II.

an emphasis on a higher grid density near the throat. This is achieved by a demand to a constant increment $\Delta \mu$ of the Mach angle between two wall points. The distribution of the Mach number along the wall is unknown, but it can be approximated by the assumption $v \approx \varphi$, where $v$ is the Prandtl-Meyer expansion angle defined as

$v(M)=\sqrt{\frac{\gamma+1}{\gamma-1}} \arctan \sqrt{\frac{\gamma-1}{\gamma+1}\left(M^{2}-1\right)}-\arctan \sqrt{M^{2}-1}$.

The shape of the nozzle in the region II is described by a function

$$
r(x)=r_{t}+\frac{x^{2}}{x_{\bar{C}}}\left(1-\frac{x}{3 x_{\bar{C}}}\right) \tan \varphi_{\bar{C}}, \quad x \in\left\langle 0, x_{\bar{C}}\right\rangle .
$$

The length $x_{\bar{C}}$ and the angle $\varphi_{\bar{C}}$ must be large enough to ensure that $M_{\text {out }}$ can be reached. It holds true, that

$$
r(0)=r_{t}, \quad r^{\prime}(0)=0, \quad r^{\prime}\left(x_{\bar{C}}\right)=\varphi_{\bar{C}} \quad \text { a } \quad r^{\prime \prime}\left(x_{\bar{C}}\right)=0
$$

and also $x_{C} \leq x_{\bar{C}}, \varphi_{C} \leq \varphi_{\bar{C}}$ which is in no contradiction to the fact, that the point $\mathrm{C}$ is not necessarily an inflection point in axisymmetric flow.

If we choose the distance $x_{\bar{C}}=x_{C}=0$ we get a socalled minimum length nozzle. The initial expansion is then reduced directly into the throat and the flow is sharply bent by an angle of $\varphi_{C}$. As follows from the theory for a $2 \mathrm{D}$ flow this angle for a planar nozzle is uniquely defined by $\varphi_{C}=v\left(M_{\text {out }}\right) / 2$. Unfortunately, for an axially symmetric flow no such analytical expression exists. The value $\varphi_{C}$ needs to be sought iteratively to achieve the equality $M_{D}=M_{\text {out }}$. According to [?] it holds $\varphi_{C}<v\left(M_{\text {out }}\right) / 4$ for $x_{C}=0$. Another difference to a planar flow is that the point $\mathrm{C}$ is not an inflection point in an axisymmetric flow.

The problems are similar in the case $x_{C}>0$. The dividing characteristic $\mathrm{CD}$ is determined iteratively such that $M_{D}=M_{\text {out }}$.

The length $x_{C}$ and the resulting overall length of the nozzle must be chosen with regard to practical requirements. In practice, the length of the nozzle is often limited by the available space or weight limits. On the other hand, the minimum length nozzle is not completely perfect in terms of the flow quality. The throat curvature is high and areas of compression can occur downstream of the curved 
sonic line. The sharp deflection of the flow in the throat leads to a production of a Prandtl-Meyer expansion fan between the first and last characteristic (i.e. Mach line). The waves are more condensed and their elimination is more geometry sensitive. In a real flow a boundary layer forms on the wall which also influences the direction of Mach lines. Any deviation from the evaluated grid causes imperfect elimination of expansion and compression waves and the disruption of the free stream. In contrast, longer nozzles, where initial expansion is more gradual, damp expansion waves by reflections from the walls and thus the sensitivity to the exact shape is reduced.

\section{Region III}

The region III is an area restricted by the right-running characteristic $\mathrm{CD}$ and the left-running characteristic DE. This part is responsible for a gradual straightening of the flow into the axial direction and formation of the homogeneous flow. The values on the left boundary are obtained as a result from the region II. The right boundary is next to the region IV where we expect a uniform, axial flow with the prescribed $M_{\text {out }}$. Therefore $\varphi=0$ and $M=M_{\text {out }}$ on DE and the slope is $\tan \mu_{\text {out }}$. The grid points are constructed from the point $\mathrm{D}$, as shown in figure 7 . The grid should be constructed beyond the point E, which position is determined by the equation (18). The resulting grid can have, for example, a pattern shown in Figure 8 (the reflected characteristics in the region II are removed from the picture).

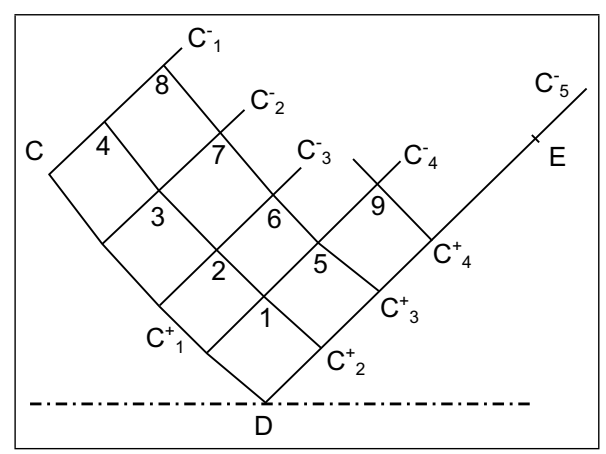

Figure 7: Creation of grid in the region III.

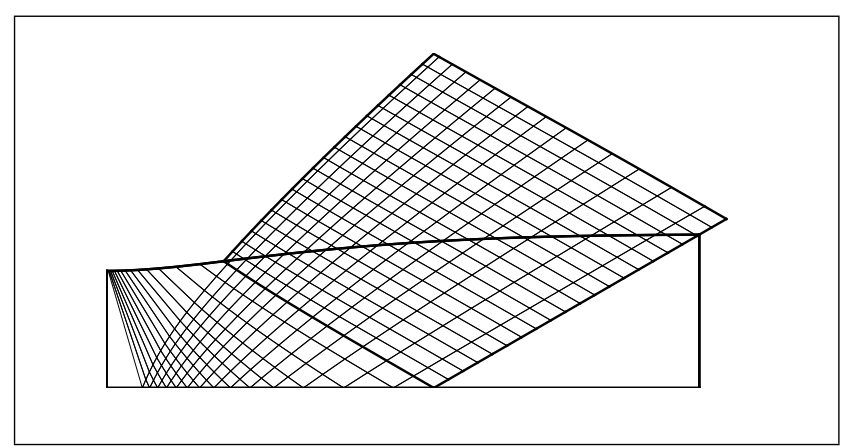

Figure 8: Complete characteristic grid in the nozzle.
After the characteristic grid is constructed and the flow values are evaluated in all nodes, the contour of the nozzle remains to be determined. The contour forming the nozzle is a streamline going through the point $\mathrm{C}$. The streamline is approximated by a piecewise linear curve that intersects the left and right characteristics. The direction of each line segment is determined by the value of the angle $\varphi$ in the intersection with a characteristic. The values in intersections are approximated by a linear combination of values in nodal points.

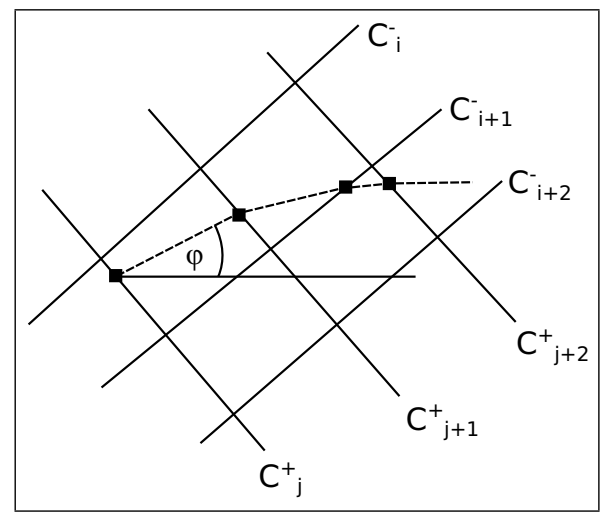

Figure 9: Construction of the streamline in region III.

The resulting contour is a piecewise linear curve which can contain some unwanted roughness, that unnecessarily disrupt the uniformity of flow. From that reason the curve is smoothed using a cubic B-spline.

\subsection{Viscous correction}

The above presented method for design of a nozzle assumes an inviscid flow. However, as was already mentioned, the real situation must deal with boundary layers which influence the effective cross-section. From this reason, the exit Mach number would be lower for such a nozzle. To overcome this problem, a correction is introduced. The correction is based on an estimate of the displacement thickness $\delta^{*}$ which broadens the nozzle geometry. This estimate is obtained with a modified Head method for a turbulent, compressible, axisymmetric flow [?].

The basis is the von Karman momentum equation in a form for compressible axisymmetric flow,

$$
\frac{d \theta}{d x}=\frac{C_{f}}{2}-\left(H+2-M_{e}^{2}\right) \frac{\theta}{u_{e}} \frac{d u_{e}}{d x}-\frac{\theta}{r} \frac{d r}{d x},
$$

with an equation for the shape parameter $H_{1}=\Delta / \theta$,

$$
\theta \frac{d H_{1}}{d x}=C_{E}-H_{1}\left(\frac{C_{f}}{2}-(H+1) \frac{\theta}{u_{e}} \frac{d u_{e}}{d x}\right) .
$$

These equations relate the momentum thickness $\theta$, mass flow thickness $\Delta=\int_{0}^{\delta} \frac{\varrho u}{\varrho_{e} u_{e}} \mathrm{~d} y$, shape parameter $H$, further the coefficients of skin friction and entrainment, $C_{f}$ and $C_{E}$, the Mach number $M_{e}$ and tangential velocity $u_{e}$ on the boundary layer edge. 
The unknown required variables $C_{f}, C_{E}$ and $H$ are evaluated from empirical relations derived from results on a flat plate with a zero pressure gradient and corrected to include the compressibility effects.

\section{Numerical verification}

\section{Subsonic flow}

The stream leaving the nozzle undergoes a homogenization over a short distance from the nozzle exit $\left(\sim r_{\text {out }}\right)$ and creates a uniform core. This core goes thinner with the increasing distance, however, at the distance $450 \mathrm{~mm}$ from the nozzle the core thickness is $50 \mathrm{~mm}$ (for $M_{\text {out }}=0.5$ ).

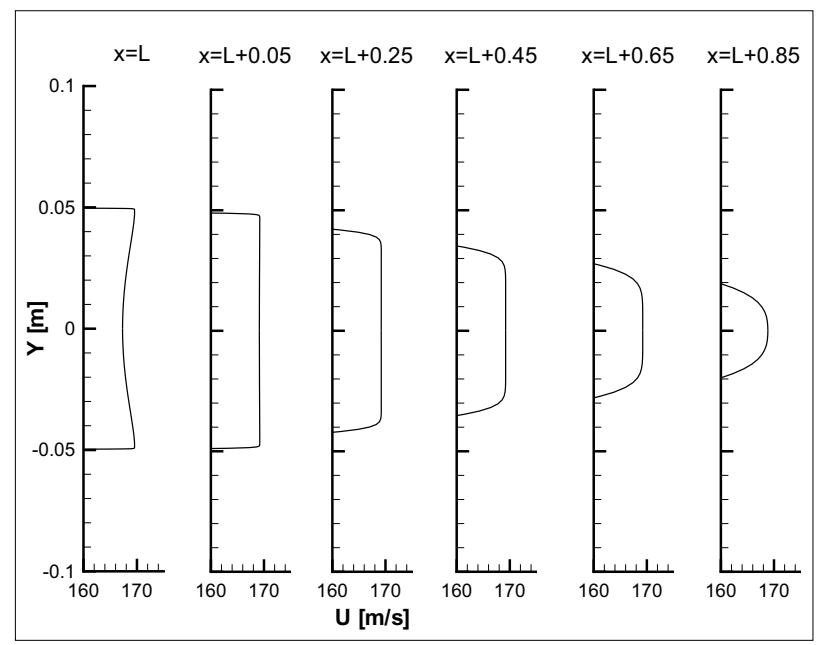

Figure 10: Velocity profiles across the stream behind the nozzle $\left(M_{\text {out }}=0.5\right)$.

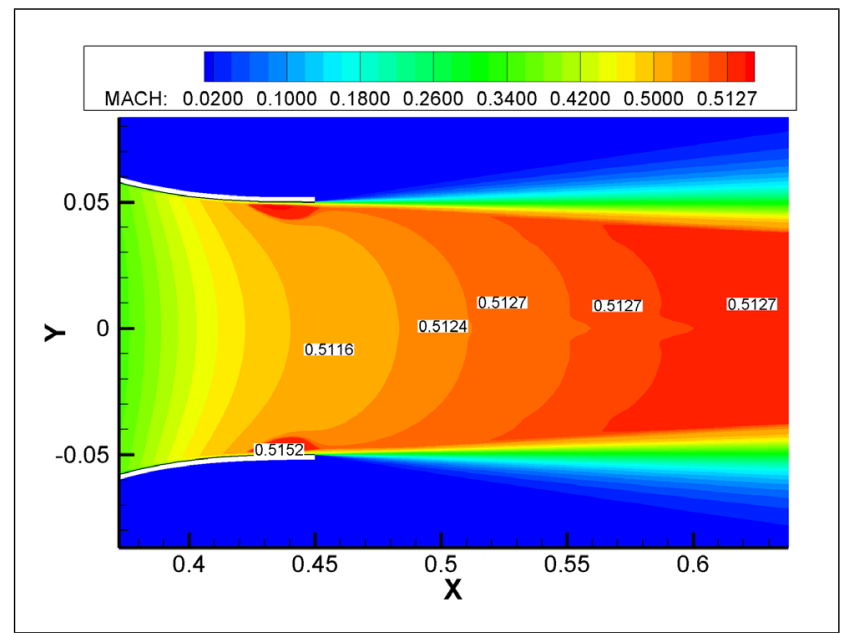

Figure 11: Mach number in the jet, higher pressure ratio due to the restricted space $\left(M_{\text {out }}=0.5\right)$.

\section{Supersonic flow}

The results confirm the expected problems for low supersonic speeds. The difference between $r_{t}$ and $r_{\text {out }}$ is very small for low supersonic Mach numbers, namely $0.2 \mathrm{~mm}$ for $M_{\text {out }}=1.1$ and $1.6 \mathrm{~mm}$ for $M_{\text {out }}=1.3$. The boundary layer thickness is $1.5 \mathrm{~mm}$ and the displacement thickness $\delta^{*}=0.3 \mathrm{~mm}$ for $M_{\text {out }}=1.3$. As a result, the Mach number is heavily dependent on the boundary layer and every imperfection of nozzle walls.

An area of a uniform flow exists behind the nozzle exit, restricted by an expansion or shock wave, which can be identified in figures $13-15$. The direction of this wave is given by the Mach angle $\mu=\arcsin \left(1 / M_{\text {out }}\right)$. In the case $p_{\text {out }}<p_{b}$ an oblique shock wave arises from the nozzle outlet and the flow is called over-expanded. If $p_{\text {out }}>p_{b}$ an expansion wave arises and the flow is called underexpanded. In the case of equality of $p_{\text {out }}$ and $p_{b}$, the design conditions are meet and a uniform flow is behind the nozzle. The figures $13-15$ show the Mach number distribution behind the nozzle in the case of an outflow into a free space.

\subsection{Influence of the testing chamber on the nozzle outflow}

The above-mentioned results were obtained as a simulation of a flow into an open space. However, the real concept includes a restricted space of the test chamber. Depending on the jet velocity, a venturi pump effect occurs which decreases the static and total pressure $p_{b}$ and $p_{b, c}$ inside the chamber. In the case of a subsonic jet, the nozzle exit velocity is higher then expected according to (2). Otherwise for supersonic regimes, the flow is under-expanded and the homogeneity of the jet is corrupted. The values of the static pressure $p_{b}$ evaluated on the outer side of the nozzle exit wall are shown in the figure 12. In reality it would be appropriate to operate with the real and actual value of $p_{b}$ and include a correction in the evaluation of the pressure ratio $p_{i n, c} / p_{b}$. The effect of the chamber is illustrated in the figure 16 .

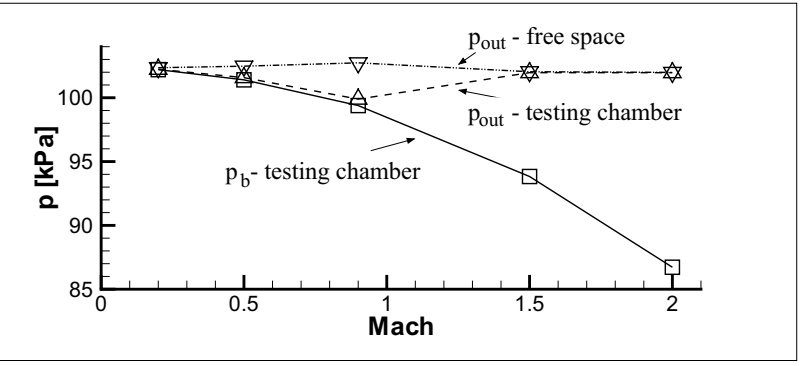

Figure 12: Effect of the restricted area behind the nozzle on the static pressure.

\section{Conclusion}

This paper presents a design of a probe calibration devise, namely the most crucial part, which is the nozzle and the testing chamber. The CFD evaluations show a suitability of the proposed solution, the resulting jet is homogeneous enough and of required parameters. The only problems 

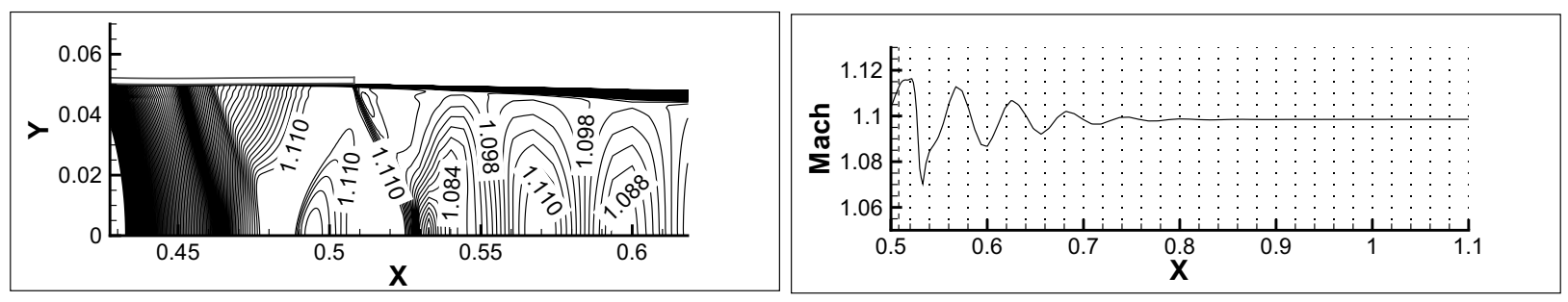

Figure 13: Mach number distribution behind a nozzle and on the axis at Mach 1.1.
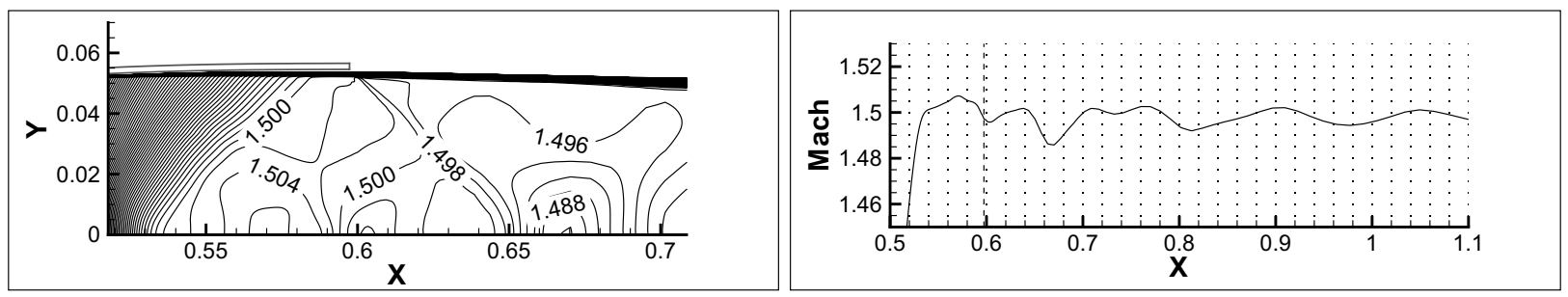

Figure 14: Mach number distribution behind a nozzle and on the axis at Mach 1.5.
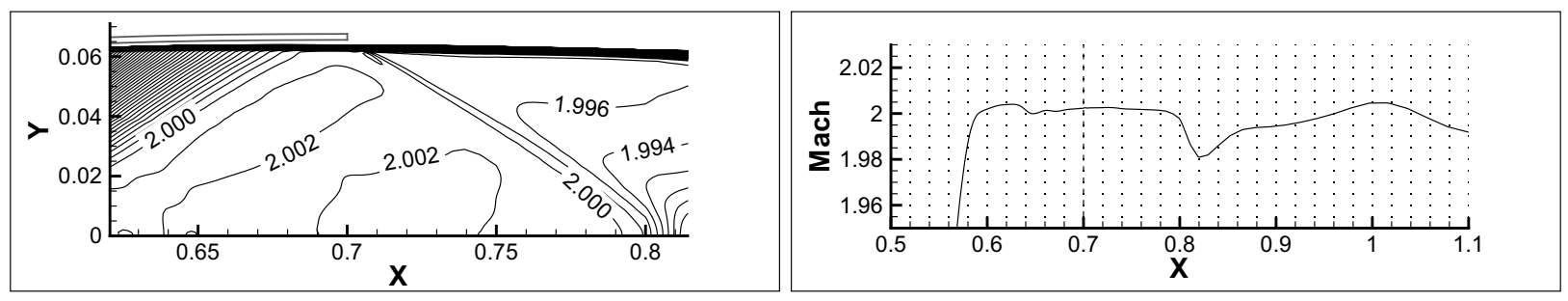

Figure 15: Mach number distribution behind a nozzle and on the axis at Mach 2.0.

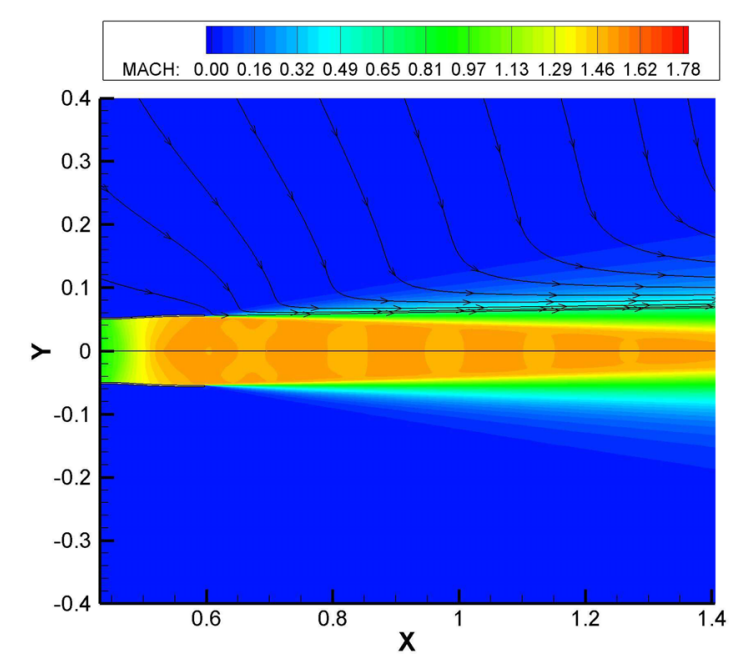

(a) free space

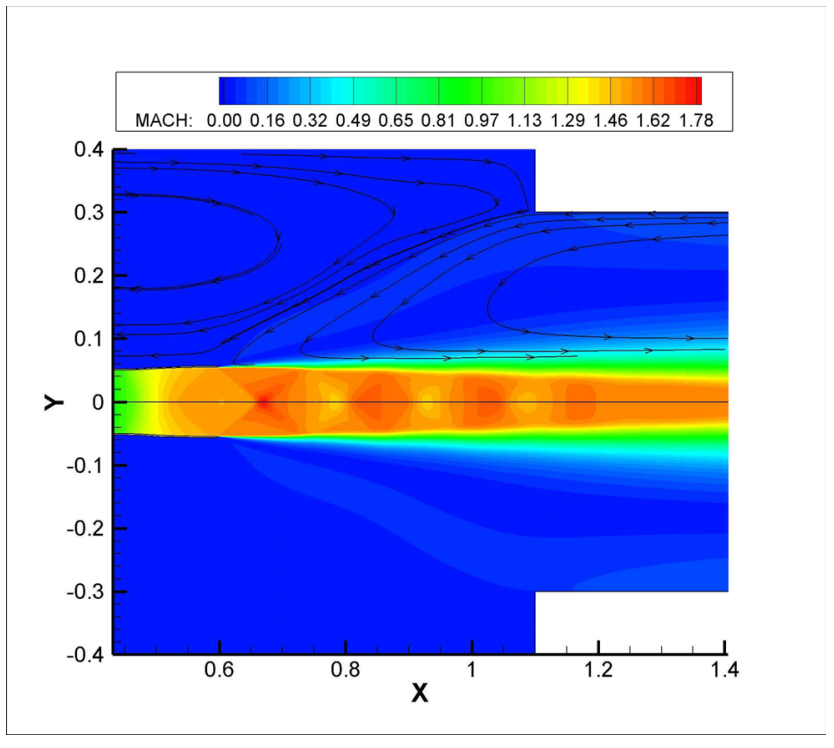

(b) test chamber

Figure 16: Effect of the test chamber on the jet $\left(M_{\text {out }}=1.5\right)$. 
could arise for low supersonic speeds when the sensitivity to the nozzle geometry can influence the outflow conditions. The jet is wide enough to accommodate various probes.

The obtained results will serve as a basis for manufacturing of the device. In the first stage, it will deal with subsonic regimes. After that, the experimental data will be compared to the numerical results.

Acknowledgement

Presented results were obtained with the support of the Ministry of Industry and Trade of the Czech Republic, aimed at a long-term development of a research organization. 